\section{Rifabutin Induced Uveitis in HIV-Dangers of Drug Interac- tions in Patients on ART}

\section{Bhargavi Pawar* and Sonika Porwal}

Department of Ophthalmology, St. Johns Medical College, Bangalore, India

\begin{abstract}
A 42 year old female, presented with sudden onset of unilateral redness, pain and blurred vision associated with photophobia and watering in the right eye of 7 days duration. She was a case of human retroviral disease on Antiretroviral Therapy (ART). Her CD4+ count at the last visit to the ART clinic was 297 . She had recently been diagnosed to have tuberculous inguinal lymphadenitis and had been started on ATT (Anti Tuberculosis Treatment) which included rifabutin, for the same. The results of her ocular examination were as follows: Visual acuity OD was counting fingers close to face, circumcorneal congestion, central DM folds, medium sized keratic precipitates, anterior chamber reaction +3 , along with a large, central dense fibrinous exudates suspended in the anterior chamber. There were exudates in the vitreous, which obscured the view of the fundus. The left eye which was normal at presentation, however, developed a similar clinical picture three days later. A review of her treatment charts revealed that she was on concurrent treatment of rifabutin and ritonavir, a combination of which is found to raise rifabutin levels in blood and produce toxic effects such as severe uveitis. The diagnosis of fibrinous rifabutin induced uveitis was made and the drug was immediately discontinued. The patient was treated with topical and oral steroids (prednisolone) for her bilateral uveitis. Rapid resolution of uveitis was followed by total visual recovery in 3 months.

Drug toxicity can occur with standard doses of $300 \mathrm{mg}$ rifabutin given as prophyaxis for Mycobacterium Avium Complex (MAC) in patients with advanced HIV disease. The effects are seen as a result of the inhibition of its enzyme related metabolism in the liver by drugs like ritonavir (component of ART), causing a rise in the blood levels of the drug. Through this case study, we wish to highlight the need for awareness, clinical suspicion and early diagnosis of drug adverse effects and interactions such as these, especially of significance in patients on ART.
\end{abstract}

\section{Introduction}

Acquired Immunodeficiency Syndrome (AIDS) in its advanced stages, leads to a susceptibility to opportunistic infections in the

*Corresponding author: Bhargavi Pawar, Department of Ophthalmology, St. Johns Medical College, Sarjapur road, Bangalore 560034, India, Tel: +91 9449833078; E-mail: dr.bhargavipawar@gmail.com

Citation: Pawar B, Porwal S (2017) Rifabutin Induced Uveitis in HIV-Dangers of Drug Interactions in Patients on ART. J Ophthalmic Clin Res 4: 026.

Received: January 17, 2017; Accepted: February 17, 2017; Published: March 03, 2017 immunocompromised host. Oral candidiasis, herpetic infections and atypical mycobacterium infections are commonly associated with AIDS. As a result of this, patients with HIV infection are commonly administered several anti microbial drugs in addition to ART.

Mycobacterium Avium Complex (MAC) infection is a common opportunistic infection in patients with Acquired Immunodeficiency Syndrome (AIDS). Rifabutin is recommended as a prophylaxis for MAC in patients with advanced AIDS is at the dosage of $300 \mathrm{mg} /$ day [1]. It is also used in the treatment of tuberculosis along with drugs like INH, pyrazinamide and ethambutol in patients with active tuberculosis. It is usually well tolerated, but is known to manifest an idiosyncratic side effect commonly seen in HIV infected individuals on Rifabutin prophylaxis - i.e., rifabutin induced uveitis [2]. It can cause anterior uveitis with or without hypopyon, intermediate uveitis or posterior uveitis with or without cystoid macular edema [3]. The onset of symptoms can occur as early as 2 weeks to 7 months following initiation of therapy [4]. It can be seen with doses that range from as low as $300 \mathrm{mg}$ a day to $1800 \mathrm{mg}$ a day [4], although side effects are less common in the standard prophylactic dose of $300 \mathrm{mg}[5,6]$. It is usually under diagnosed, and the patient is subjected to various laboratory tests to establish an etiology for the uveitis. Since the number of HIV infected patients all over the world is significant, clinicians need to be aware of the incidence and the consequences of HIV related conditions.

\section{Case Details}

A 42 year old female was referred to our ophthalmology department with complaints of sudden onset of redness, pain and blurring of vision in the right eye of 1 week duration. It was associated with photophobia and watering. The patient was a known case of retroviral disease diagnosed in 2003 and had been on Antiretroviral Therapy (ART) since then. Her drug regimen included tenofovir $300 \mathrm{mg}$, lamivudine $300 \mathrm{mg}$ atazanavir $300 \mathrm{mg}$ and ritonavir $100 \mathrm{mg}$. She had a history of Pneumocystis Carinii Pneumonia (PCP) and Herpes labialis infection a year earlier and was treated for the same, and was continued on PCP prophylaxis of sulphadiazine and pyrimethamine in a fixed drug combination. She was diagnosed to have tuberculous inguinal lymphadenitis a month prior to the onset of ocular symptoms and had been started on Anti Tuberculosis Treatment (ATT), which included INH $300 \mathrm{mg}$, pyrazinamide $15 \mathrm{mg} / \mathrm{KGB}$, ethambutol $800 \mathrm{mg}$ and rifabutin $300 \mathrm{mg}$.

Ocular examination revealed the following: Best corrected visual acuity OD -Finger Counting (FC) at $1 / 2$ metre and BCVA OS - 6/6. Anterior segment examination showed circumcorneal congestion, central Descement's membrane folds, medium sized keratic precipitates, normal anterior chamber depth with a $3+$ reaction and a central dense coagulated fibrinous exudate with a $2 \mathrm{~mm}$ hypopyon (Figure 1)

Fundoscopy through a hazy media showed exudates in the vitreous cavity (Figure 2)

The left eye anterior segment and fundus was normal at presentation, but developed an identical clinical picture within 3 days of the right eye manifestations. 


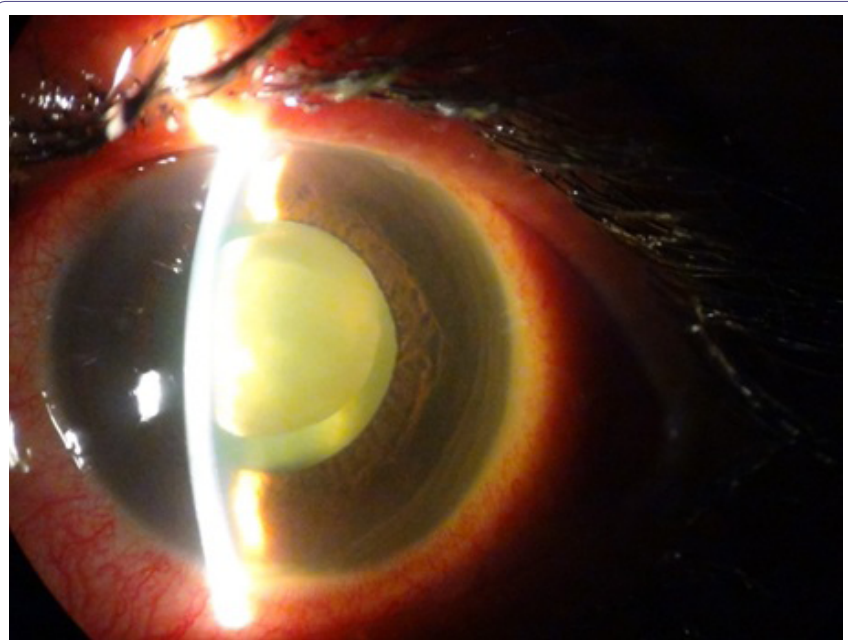

Figure 1: Dense Fibrinous exudates in the anterior chamber mimicking a lens nucleus.

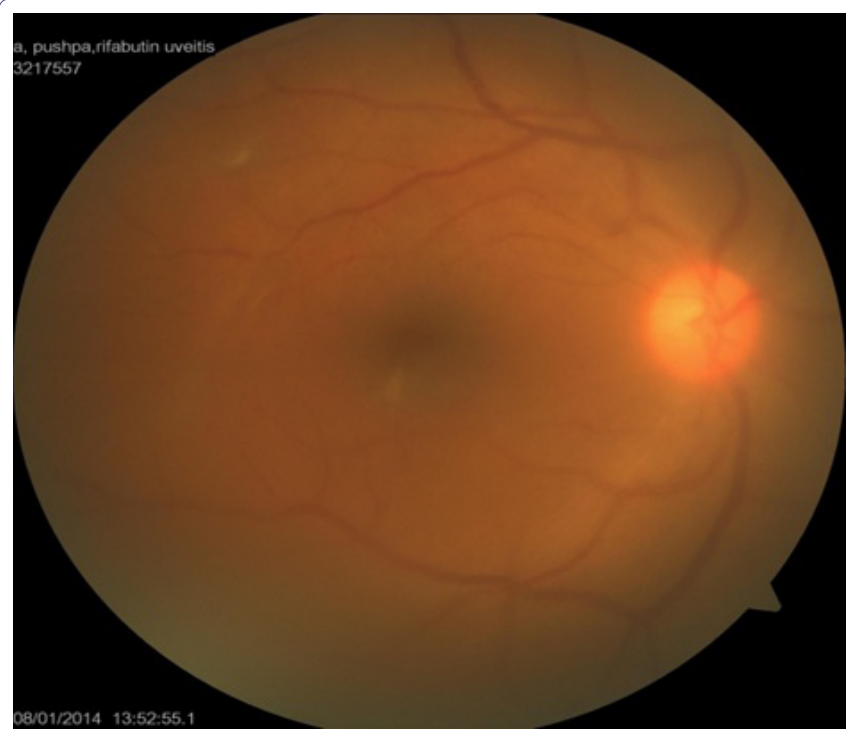

Figure 2: Hazy view of the fundus due to vitreous exudates.

Her blood tests results were as follows : Hemoglobin 9.9g, Total count 8.940 cells/cu.mm, differential count ( N70 L25 M4 E1 B0) normocytic normochromic peripheral smear, CD $4+=297, \mathrm{CD} 3+=$ $1064, \mathrm{CD} 45+=2126$.

After reviewing her treatment charts, a diagnosis of rifabutin induced uveitis was made and the patient was started on oral prednisolone $40 \mathrm{mg}$ and topical steroids (Prednisolone acetate $1 \%$ hourly ) and topical cycloplegics (atropine sulphate $1 \%$ twice daily). Immediate discontinuation of rifabutin therapy was suggested after a physician consultation. The INH, prazinamide and ethambutol were however continued. The oral and topical steroid therapy for the uveitis was slow tapered over a 3 month period. Rapid resolution of the bilateral uveitis was observed (Figure 3,4 ) within a fortnight and was followed by total visual recovery in 3 months.

\section{Discussion}

Rifabutin associated uveitis has been described in AIDS patients and is identified as a dose-dependent, idiosyncratic side effect [7]. Adverse effects are unusual at the recommended dose of $300 \mathrm{mg} /$ day [8],

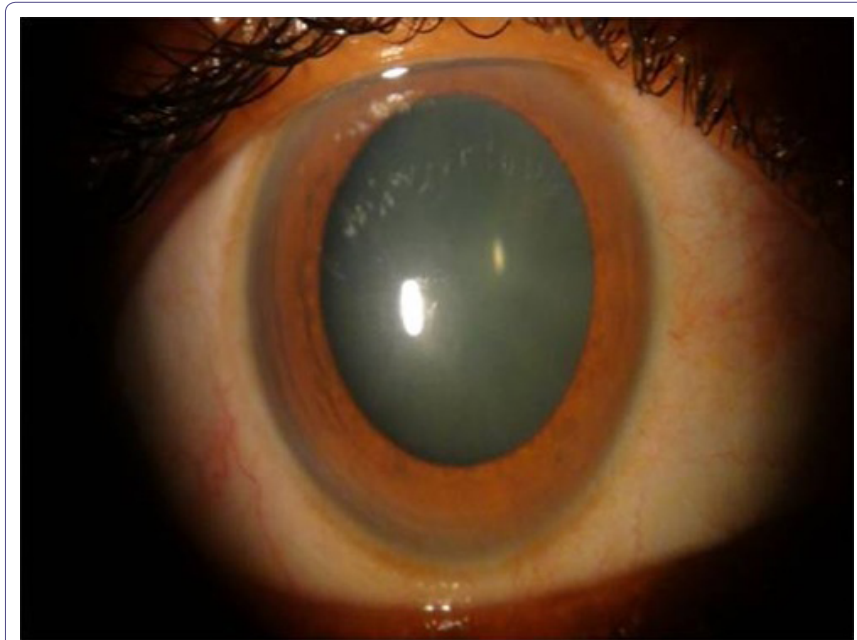

Figure 3: Complete resolution following steroid therapy and cessation of rifabutin (OD).

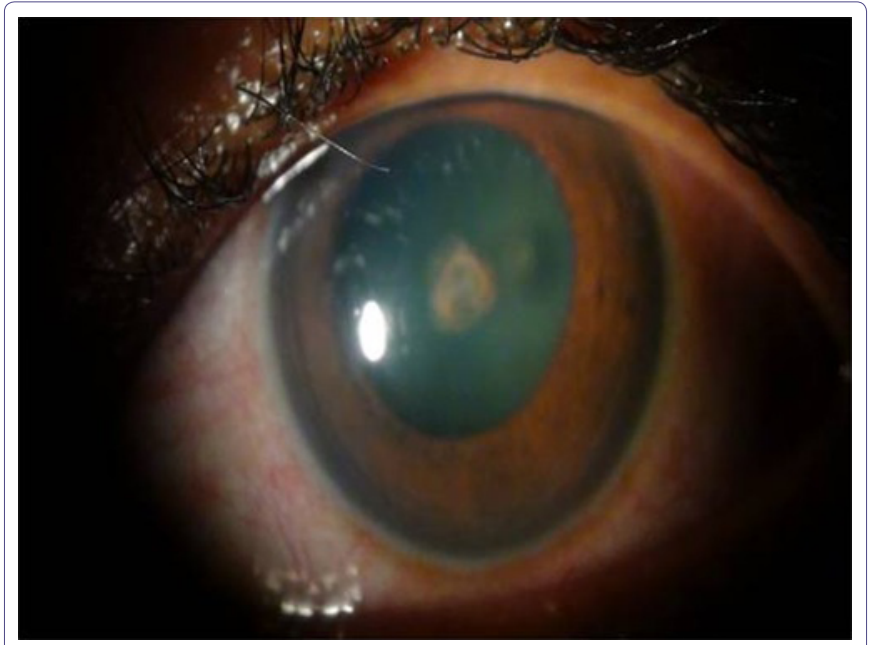

Figure 4: Resolving anterior uveitis in the fellow eye (OS).

however, it can be seen with doses that range from as low as $300 \mathrm{mg}$ a day to higher doses of $1800 \mathrm{mg}$ a day [4].

Ritonavir (a component of ART) is a potent inhibitor of CYP3A4, a liver enzyme responsible for drug metabolism, and has been shown to substantially increase Rifabutin concentration in blood by indirectly inhibiting its metabolism.

Co-administration of rifabutin with ritonavir, as is common in patients with HIV disease, increases the Area Under the Concentration-time curve (AUC) of Rifabutin by 35 times, compared with administration of rifabutin alone $[5,6]$. This increases the propensity for rifabutin to exert its ocular idiosyncratic effect. It has also been associated with concomitant use of clarithromycin or fluconazole, which is believed to raise serum levels through inhibition of the hepatic cytochrome p-450 system that metabolizes the drug [9].

It has also been described in immunocompetent indivuals with atypical mycobacterial infections [10]. Studies conducted by Havlir et al. [11] and Kelleher et al. [12] have shown that the interval between starting rifabutin and the onset of uveitis was 7 months and 2 months respectively.

The dose required to cause uveitis could range anywhere from $300 \mathrm{mg}$ to $1800 \mathrm{mg}$ as established by Skolik et al. [13], The higher the 
dose and duration of treatment, the greater is the risk of associated uveitis [14]. Our patient developed uveitis within one month of initiation of therapy at a standard dose of $300 \mathrm{mg}$, the early onset of the drug side effect is attributable to the inhibition of its metabolism by ritonavir.

The treatment is aimed at stopping the offending agent along with anti inflammatory and immunosuppressive therapy to help the inflamed uveal tissue recover. Discontinuation of rifabutin with concomitant use of topical and a short course of systemic steroids is effective in reversal of the condition. The importance of reviewing treatment charts and awareness of drug interactions in cases such as these would obviate the need for unnecessary and extensive investigations that patients of uveitis are usually subjected to. An early suspicion of rifabutin induced uveitis could also prevent invasive ocular procedures like aqueous sampling and a detailed drug history should be elicited in unexplained uveitis [15]. In our patient, rapid resolution of uveitis (Figures 3 and 4) was followed by total visual recovery within 3 months time. No invasive diagnostic procedure was carried out to confirm the diagnosis, although it is recommended to consider a blood sampling to determine serum rifabutin levels in patients with uveitis.

\section{Conclusion}

We wish to highlight the need for awareness, clinical suspicion and early diagnosis of drug adverse effects and interactions such as these in patients on ART. Doctors who prescribe rifabutin should be aware of this ocular complication of uveitis and drug interactions related to Antiretroviral Therapy (ART). General ophthalmologists and specialists in uveitis must have a suspicion for drug induced uveitis in their list of differential diagnoses.

\section{References}

1. CDC (1993) Recommendations on prophylaxis and therapy for disseminated Mycobacterium avium complex for adults and adolescents infected with human immunodeficiency virus. MMWR, CDC, Atlanta, USA. Pg no: 42: 14-20.

2. Saito T, Oban A, Tsuchiya $Y$, Saito K, Hotta $Y$ (2011) [Three cases of uveitis induced by mycobacteriosis therapy using rifabutin]. Nippon Ganka Gakka Zasshi 115: 595-601.
3. Vaudaux JD, Guex-Crosier Y (2002) Rifabutin induced cystoids macular oedema. Journal of Antimicrob Chemother 49: 421-422.

4. Tseng AL, Walmsley SL (1995) Rifabutin-associated uveitis. Ann Pharmacother 29: 1149-1155.

5. Skinner MH, Blaschke TF (1995) Clinical pharmacokinetics of rifabutin. Clin Pharmacokinet 28: 115-125.

6. Cato A 3rd, Cavanaugh J, Shi H, Hsu A, Leonard J, et al. (1998) The effect of multiple doses of ritonavir on the pharmacokinetics of rifabutin. Clin Pharmacol Ther 63: 414-421.

7. Nightingale SD, Cameron DW, Gordin FM, Sullam PM, Cohn DL, et al. (1993) Two controlled trials of rifabutin prophylaxis against Mycobacterium avium complex infection in AIDS. N Engl J Med 329: 828-333.

8. Saran BR, Maquire AM, Nichols C, Frank I, Hertle RW, et al. (1994) Hypopyon uveitis in patients with acquired immunodeficiency syndrome treated for systemic Mycobacterium avium complex infection with rifabutin. Arch Ophthalmol 112: 1159-1165.

9. Moorthy RS, Valluri S, Jampol LM (1998) Drug-induced uveitis. Surv Ophthalmol 42: 557-570.

10. Jetton J, Gaviria JG, Patterson TF, Johnson DA (2009) Rifabutin-associated uveitis in an immunocompetent individual with Mycobacterium simiae. Can J of Ophthalmol 44: 468-469.

11. Havlir D, Torriani F, Dubé M (1994) Uveitis associated with rifabutin prophylaxis. Ann Intern Med 121: 510-512.

12. Kelleher P, Helbert M, Sweeney J, Anderson J, Parkin J, et al. (1996) Uveitis associated with rifabutin and macrolide therapy for Mycobacterium Avium Intercellulare infection in AIDS patients. Genitourin Med 72: 419-421.

13. Skolik S, Willermain F, Caspers LE (2005) Rifabutin-associated panuveitis with retinal vasculitis in pulmonary tuberculosis. Ocul Immunol Inflamm 13: 483-485.

14. Cordero-Coma M, Salazar-Méndez R, Garzo-García I, Yilmaz T (2015) Drug-induced uveitis. Expert Opin Drug Saf 14: 111-126.

15. London NJ, Garg SJ, Moorthy RS, Cunningham ET (2013) Drug-induced uveitis. J Ophthalmic Inflamm Infect 3: 43. 